\title{
Supporting Conceptual Design: A Model for Reflective-Interactive Design
}

\author{
Bill Chan ${ }^{1} \&$ Susan Finger ${ }^{2}$ \\ ${ }^{I}$ Visionary Design Systems, Atlanta GA, USA \\ ${ }^{2}$ Carnegie Mellon University, Pittsburgh, PA, USA
}

Key words: conceptual design, computer-aided design, knowledge-intensive CAD

\begin{abstract}
In the process of developing a concept, a designer will view a design from multiple levels of abstraction, using mental simulation to verify the interaction between its different components. This interaction can be viewed as a conversation between the design and the designer, which is conducted in the languages of function, behaviour and form. A computational design tool that supports this conversation will both enrich the quality of the interaction, as well as provide a mechanism to capture the knowledge embedded within the discourse. Designers generate knowledge about aspects of the design artefact, other than its physical attributes, as part of the process in early design. This knowledge is valuable because it establishes the design context. Understanding of context is critical to guide downstream design processes such as model abstraction and evaluation. In this paper, we present a framework and initial computer implementation of a reflective-interactive design methodology. Using this framework, a designer can create and simulate incomplete and evolving designs using function, behaviour, and form concepts.
\end{abstract}

\section{INTRODUCTION}

The process of design is not function-driven per se; nor is it purely behaviour- or form-driven. In exploring possible design solutions, a designer tries to achieve function through some intended behaviour. Form acts to both enable and constrain the actual behaviour. Simulation of the design determines whether the actual behaviour is the same as the intended 
behaviour. The actual behaviours of complex systems are not known with certainty by the designer $a$ priori and must be verified using virtual or physical prototypes.

Designers often use mental simulation to explore ideas at an early stage in their development, before details about structure or material properties have been defined. They use mental simulation to gain understanding the interaction between different parts of a design and between the design and the surrounding world. Yet predicting behaviour of complex systems mentally is often difficult and error prone. Computer simulation can be used to analyse the behaviour of completed designs; however, these simulations tend to be expensive, both in terms of the computational power and time needed to run the analysis, as well as in terms of the amount of time and effort needed to create a computable model of the design. For this reason, computational simulation is rarely used the way humans routinely use mental simulation.

We believe that the usefulness of such simulation is not limited to its analytical value. Rather, simulation is a mechanism through which the designer interacts with the design and explores the design space. It provides feedback to the designer about the consequences of a design move, and therefore increases the designer's understanding of the design problem. We believe that this interaction is an important aspect of the conversation between design and designer, which promotes reflection by the designer. A computational environment that allows the designer to interactively construct and simulate incomplete models of a design would enrich the conversation between the designer and the design.

Design knowledge created during the early stages of design can be used to advantage in the later stages of design. The task of capturing and organizing this design information should take place as part of the design process, not as a post-process after the design is complete. Design methods and tools need to be synchronized with the technical and social requirements of the design environment (Baya and Leifer, 1994). Rather than having separate models of a design during conceptual design and embodiment design, a single representation should provide continuity in the flow of design knowledge between the different stages of the overall process. A single design data-model should support the refinement of high-level abstractions of function and behaviour, through the addition of general characteristics of form during embodiment design, to the detailed specification of all its physical attributes in detailed design.

Existing CAD tools are not well suited for conceptual design because their functionality retains much from their origins in drafting and electronic storage of design geometry. Some proposed conceptual design tools have taken an analytical approach based on a systematic decomposition of an 
initial problem description. Others seek to automatically generate design solutions or to automate the exploration of the space of possible designs. Neither of these approaches is suitable for activities that are intrinsically synthetic and exploratory in nature. The lack of CAD support during early design isolates the early design activities from the later engineering activities and restricts the transmission of design knowledge between the different stages of the design cycle. Specifically, valuable design knowledge generated by the designer during early design is never captured for use in downstream processes.

\section{REFLECTIVE-INTERACTIVE DESIGN}

An important aspect of our work is the idea of design as reflective conversation. The phrase, "reflective conversation" was coined by Schön (Schön, 1983) in the larger context of reflection-in-action; his proposal for an alternative philosophy of practice. Reflection-in-action was Schön's response to what he felt were negative trends in professional practice that derived from ideas championed under the paradigm of Technical Rationality.

The reflective-interactive view of design holds implications for the practice of design and the tools that support it. Current prescriptive methodologies for design describe a process in which the designer acts on a design, effecting changes on a static representation without accounting for the designer's perception and reactions to the results of his actions. For example, simulation of a design's behaviour can expose problems that were not known beforehand or can provide insight to the designer about the design problem. This new knowledge can change the designer's formulation of the design requirements. Reflective-interactive design emphasizes the importance of the interaction that occurs between the designer and the design situation. The design situation includes the design and its relationship to the surrounding environment. Thus, the design environment must be able to communicate the effect that design moves have on the behaviour of the design and that the design representation support this functionality.

Based on the reflective practice model, our reflective-interactive model expands the scope of design to include the designer's situation, in addition to the design process oriented view taken in the rational problem-solving paradigm. In particular, the reflective-interactive view builds on the idea of reflective conversation as a metaphor for the mechanism whereby the designer interacts with the design situation.

Design methods and techniques must be built on three supporting structures: a model of the designer, a model of the design task, and a model 
of the dynamics of the design process. These three classes of models respectively define: the cognitive processes or mechanisms of action of the designer; the structure and methods within a particular design task; and the meta-level control structures and processes of design. In his comparison of rational problem solving and reflective practice, (Dorst and Dijkhuis, 1995) summarizes these three aspects for both paradigms. In the rational problemsolving paradigm, the designer is modelled as a finite-state machine, the design task is modelled as an ill-structured problem, and the design process is modelled as a generalized search. Under the theory of reflective practice, the designer is modelled as a person who constructs his or her own understanding of the world based on his or her perceptions. Each design task is essentially a unique construction of the designer, and the process of design is a reflective conversation with the situation.

The early stages of design involve a process of exploration in which the designer refines his or her understanding of the design problem through reflection and interaction with virtual and physical models of design concepts. Some researchers refer to this process as a meta-level search of the design space with the goal of obtaining knowledge for guiding further searches within the design solution space (Smithers and Troxell, 1990, Gero and Kazakov, 1996, Poon and Maher, 1997). Other use exploration in a purely generative manner (Faltings, 1992; Tanir et al., 1993).

Knowledge about aspects of the design artefact, other than its physical attributes, is generated as part of the process in early design. This knowledge is valuable because it establishes the design context, and an understanding of context is critical in guiding downstream design processes such as model abstraction and evaluation. Support for this claim can be found in the recent literature. The work of (Allen, 1989, Tomiyama et al., 1989, Ganeshan et al., 1992, Subrahmanian et al., 1993, Reddy, 1996, SEED, 1997) each include the idea that design creates and uses many different types of knowledge. To provide additional experimental support for this claim, we have analysed the content of design discourse between members of a team of six designers to determine the nature of the information that is created and used as part of designing. The results of this analysis are reported in (Chan, 1998, Reddy et al., 1996).

A computational design tool that supports a reflective-interactive model of design provides an opportunity to capture the artefact knowledge that describes design context. The representation of the design model should also support the evolutionary refinement of the design after the early stages of design so that any additional knowledge generated is also captured. Existing and proposed product data-models are meant to capture product information for completed designs (Shaw et al., 1989). 


\section{CHARACTERIZING DESIGN ARTIFACT INFORMATION}

The knowledge that is created and used in the course of design can be differentiated as being concerned with the design process or the design artefact. Design process knowledge relates to the methods and practice of the design process. Examples of process knowledge include: the history of design decisions, the best formulation of a mathematical model for a class of problems, or a list of suppliers for a particular part. Design artefact knowledge relates specifically to the attributes, structure, and qualities of the artefact being designed. Within this research, we will only be concerned with knowledge about the design artefact that relates to design intent.

We partition the space of information about a design artefact into phases. The term "phase" is used exclusively in its chemical sense to identify a distinct region within a mixture, and not in its temporal sense. The process of design populates the solution space with information and organizes this information into the knowledge that defines the design intent of an artefact.

We use two basic structures to describe design artefacts: concepts and relationships. Because we are concerned with mechanical design, we assume that all concepts will be realized through physical means. Therefore, concepts possess physical form. Concepts can correspond to an entire design artefact, components in the artefact, parts of components, or regions of a part. In the early stages of design, however, a designer may think of a concept as an abstract entity without physical form. In this case, a concept is given form as part of the design process, through a process of refinement in which the design is fleshed-out. For example, a functional requirement that the height of an office chair seat be adjustable vertically can be instantiated as a behaviour where the seat translates in the vertical direction relative to the base of the chair. This behaviour links the motion of the chair seat with the position of the base. However, the specific geometry of a mechanism to achieve this behaviour can take on several different physical forms (e.g., screw mechanism, four-bar linkage, rack and pinion, prismatic joint, etc.). Allowing the designer to defer commitment to a specific form until a later time in the design process ensures that the design solution space is not restricted before the designer has an opportunity to properly explore solution concepts. Most existing product models implicitly include information about form within the basic entity used to construct a design model. For instance, the design entity used with the SHADES framework (Hakim, 1993) includes knowledge about at least two design aspects (phases in our terminology). 


\subsection{Function, Behaviour and Form}

In our research, we differentiate between function and behaviour. We define function to be the intended use of something. In the context of representation of design knowledge, function can be thought of as design intent. Behaviour is the manner in which something acts or reacts in the physical sense. Form refers to the geometry of a design as well as the physical properties of the material from which it is constructed. A common problem in design occurs when the actual behaviour of a design does not match its intended behaviour. Alternatively, an unexpected secondary behaviour might emerge in a design that violates an initial design requirement. In both cases, the design will not achieve its function.

Function encodes high-level knowledge about goal and context for an artefact. Behaviour complements functional information by defining the "how" for a design. Knowing what a design is supposed to do does not tell us how that goal is to be achieved. Knowledge about the behaviour of an artefact can be used to determine the interactions between parts of a design.

\subsection{Concepts}

Concepts are composed of elements from three phases in the design artefact information space. Each phase relates to a different aspect of the concept: its function, its behaviour or its form. Therefore, a concept, like the overall information space, is composed of three phases, each of which exists within the concept as a distinct part of the concept. Phases do not mix with one-another, however the state of one phase may have an effect on another phase. Therefore, relationships may exist between entities in different phases, as well as between entities within a phase. Some of the relationships within a phase, such as the part-of relationship, serve to define a hierarchy of abstraction within that phase. Relationships may also be topological (e.g., adjacent-to) or causal (e.g., enables) in nature. Figure 1 below illustrates the structure of design artefact information space, and the relationship between the phases within the total information space, and a single concept. 


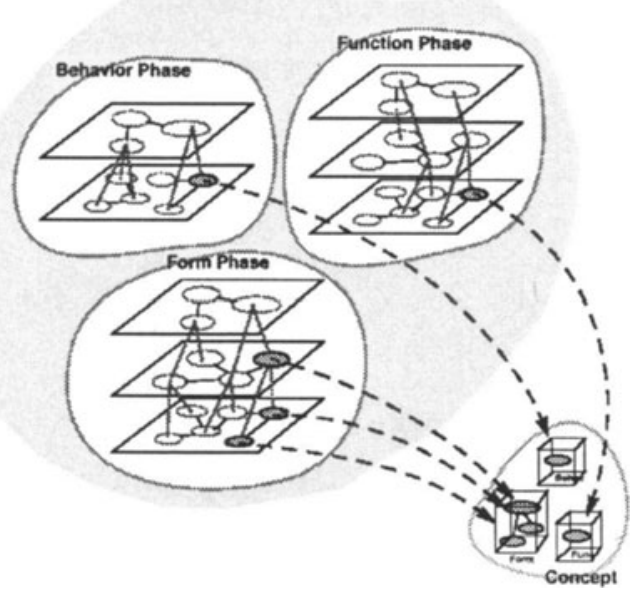

Figure 1. The structure of design artefact information space

\subsection{Descriptors}

Concepts are described by parameters or more generally, descriptors. Descriptors are typically associated with a particular phase. Therefore, descriptors describe a concept from the perspective of function, behaviour or form. The relationships among concepts are established between the concepts' descriptors. Relationships acting between descriptors in a phase relate concepts to one another and in this manner create the conceptrelationship network that defines the design intent.

Each phase type has its own descriptors. Descriptors for the form phase include the geometric and material attributes inherent to the design artefact. Examples include geometric parameters such as length and width; topological primitives like face, edge, or vertex; and material attributes like density and Young's Modulus. Descriptors for the behaviour phase are derived attributes that involve an external disturbance acting on the concept. Examples of this type of descriptor include weight, motion, deflection, etc. Descriptors for the function phase describe the intended use of the concept and include machine element types such as lever, gear, bearing-surface, etc. Table 1 shows examples of descriptors for the three phases. 
Table 1. Examples of descriptors for function, behaviour, and form phases

\begin{tabular}{|c|c|c|}
\hline Form Phase & Behavioural Phase & Functional Phase \\
\hline $\begin{array}{l}\text { Inherent attributes } \\
\text { - length, diameter } \\
\text { - } \text { face, edge, vertex } \\
\text { - } \quad \text { density, Young's } \\
\text { Modulus }\end{array}$ & $\begin{array}{l}\text { Derived attributes } \\
\text { - weight } \\
\text { - motion } \\
\text { - deformation, } \\
\text { temperature }\end{array}$ & $\begin{array}{l}\text { Intended use } \\
\text { - lever } \\
\text { - gear } \\
\text { - bearing-surface }\end{array}$ \\
\hline
\end{tabular}

\subsection{Concept Structure}

The concept structure is the basic building element used in the synthesis of a design solution. A concept entity can be thought of as a design feature in that it identifies a part or region of a design that has special significance to the designer. The specific nature of this significance is dependent on the context in which the design is examined. For instance, a concept might have significance related to its performance or ability to achieve its design functions. Examples of this type of design significance might include: resistance to plastic deformation under an applied load, rate of heat dissipation, or motion due to an applied force. Alternatively, a concept might be significant from the perspective of downstream considerations like manufacturing or assembly. Examples of concepts that are explicitly significant to downstream processes include locating-pins and bosses. A designer might create these features explicitly for the purposes of the downstream assembly process. More problematic are features related to downstream constraints that are emergent. These are features that are not explicitly created for downstream processes but rather emerge from the form associated with an existing concept or combination of concepts. Examples of emergent features can include undercuts or thin-walled sections that may pose problems for machining processes. The designer may not be able to recognize this type of feature a priori.

Since geometry and material properties are simply one aspect of a general concept entity (specifically that of form), and the operations of constructing geometric models and assigning material properties as instantiating relationships between concept entities, then the typical formdriven method of design can be understood as a process of instantiating and structuring concept entities. In our model of artefact information, a concept can be related to other concepts via relationships that act within one phase of the design artefact information space, as well as through relationships that act across different phases. In terms of its physical form, a concept is represented by its relationships within the form phase. 


\subsection{Relationships}

Design is a process of generating knowledge. From an initial body of design artefact information, which might include design requirements and constraints, the designer creates new instances of artefact information and defines relationships between these information structures, to create knowledge about the design artefact. There are two types of relationships: inter-phasic and intra-phasic. While inter-phasic relationships only act within a concept, intra-phasic relationships exist both within concepts as well as across concept boundaries. An example of an inter-phasic relationship between the functional and behavioural phases would be several behaviours that combine to achieve a single function. An example of an intra-phasic relationship within a single concept would be several subfunctions that combine to create an overall function. An example of an intra-phasic relationship acting between two concepts would be the causal effect of the behaviour of one concept on the behaviour of another concept.

Figure 2 illustrates how inter- and intra-phasic relationships can be used to define more complex concept structures. In the example shown below, Concept- 1 and Concept- 2 are linked by two intra-phasic relationships, R1 and R2. Similar relationships are shown between Concept-1 and Concept-3. $\mathrm{R} 1$ is a causal relationship between a behaviour in Concept- 1 and a behaviour in Concept-2, and R2 is a topological relationship between the two concepts' respective physical embodiments. In addition, similar relationships can be defined between Concept-1 and Concept-3. The resulting set of concepts linked by relationships creates a network. One interpretation for the concept-relationship network described is that of two linkages (Concept-2 and Concept-3) connected by a pin joint (Concept-1). Figure 3 depicts a possible physical embodiment for two simple linkages connected by a pin joint. 


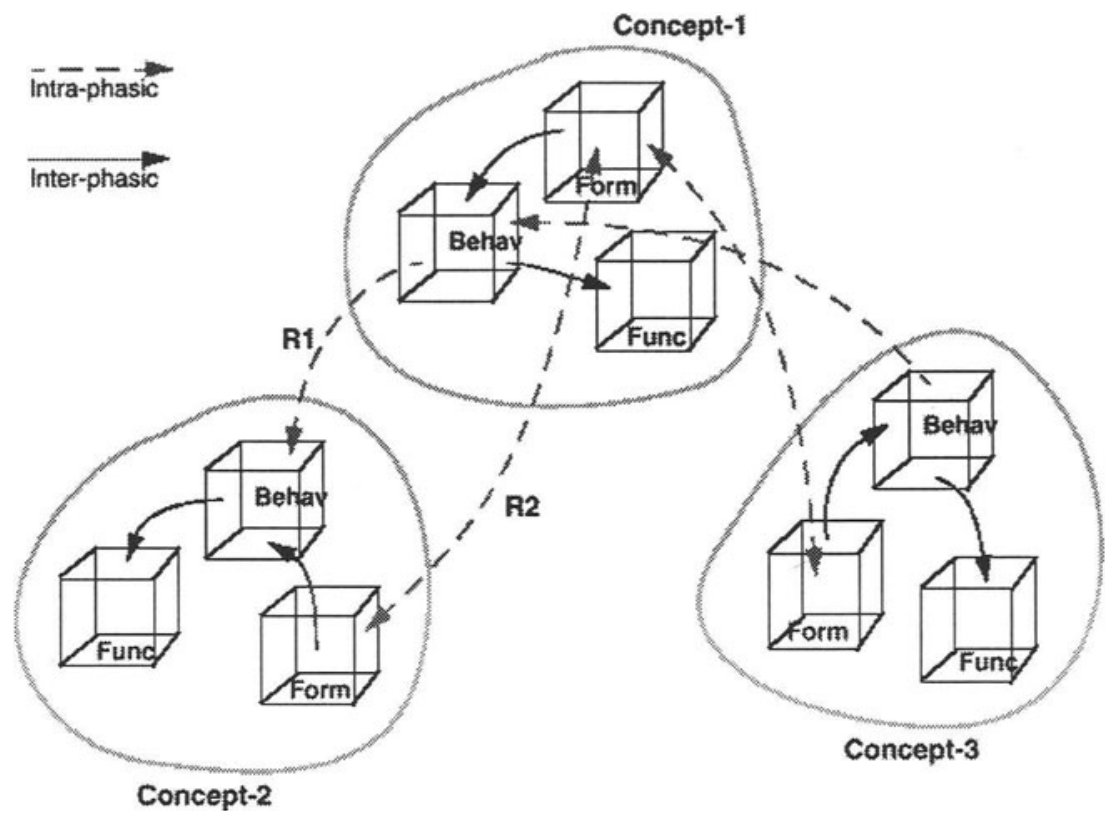

Figure 2. Intra-phasic relationships act between concepts as well as within concepts, while inter-phasic relationships act only within concepts

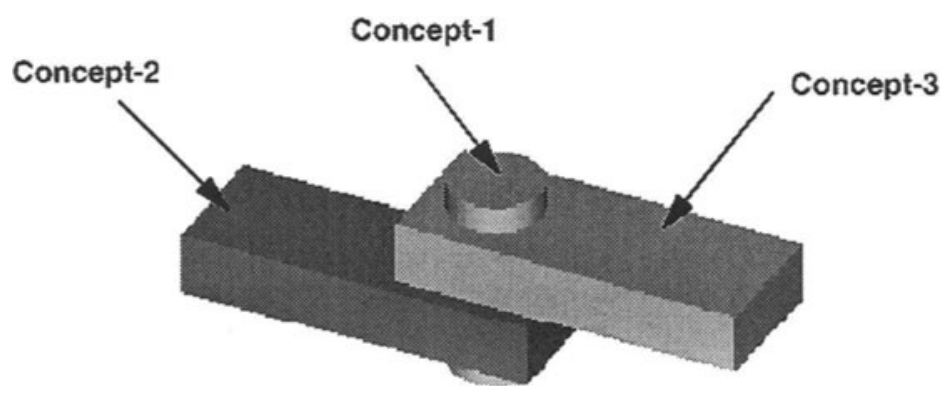

Figure 3. Two linkage arms connected with a pin joint, interpreted in terms of the conceptrelationship network in Figure 2

The designer generates a concept to address a particular design requirement or constraint, and embeds the new concept within the evolving design context by defining its relationships to other concepts. As the design process proceeds, the designer refines and modifies the concept-relationship network, adding detail to concept descriptions and relationships. We have 
found that designers generate and work with concepts in a heterogeneous manner, in terms of function and behaviour, as well as form. These three aspects of the design are sub-sets of the information contained within three distinct phases of the total space of design artefact information. The relationships instantiated as part of design create a network of concepts across the different phases, as well as a hierarchy of abstraction within each phase.

\section{IMPLEMENTATION}

The architecture for our computational environment is built around a design modelling kernel, which plays the same role within the design environment as the solid-modelling kernel does within many existing CAD systems. The primary difference between the two kernels is that solidmodelling kernels are meant to model the geometry and topology of solids, whereas our design modelling kernel extends the capabilities of the solidmodelling kernel to model the knowledge about functional and behavioural intent that sets the context of a design. Simulation capabilities are provided by external modules that access the design data-model through its interfaces. For the initial prototype, only two interfaces are provided: a direct interface to the data-model through the public data members and methods of the object classes, and a formal application protocol interface.

\subsection{The Modelling Kernel}

The design modelling kernel provides a set of core object classes and interfaces to the data and methods within these classes. The object classes within the design modelling kernel include those that one would expect to find in a solid-modelling kernel (e.g., object classes such as body, shell, edges, vertex, sphere, plane, and point). These topological and geometric classes provide the capabilities needed to describe the design in terms of its form phase, as well as the basic geometric functionality of the modelling kernel. The design-modelling kernel extends the solid-modelling capabilities with the addition of object classes for composing the functional and behavioural knowledge structures that define the intent behind the physical embodiment of the design.

In our prototype implementation, we use the ACIS solid-modelling kernel by Spatial Technologies, Inc. (STI, 1997). ACIS is based on a nonmanifold boundary representation. We use ACIS's ability to represent bodies of mixed dimensionality to provide the capability of modelling the 
abstract knowledge structures alongside the physical form. Furthermore, the ability to represent non-manifold bodies is a requirement for a modelling environment that supports design at heterogeneous levels of abstraction.

A three-dimensional graphical interface for the design modelling environment is needed to support an interactive design experience that promotes exploration of design concepts. The current 3D mouse-based interface allows the designer to construct the design model within a GUI framework similar to the interface for existing 3D solid modellers. Standard views such as isometric and the different orthographic projections are provided, along with various camera tools for zooming, panning, and orbit. The design model can be either viewed as a wireframe or rendered object. (See Figure 4.) OpenGL provides the basic rendering functions (Woo et al., 1997). The interface also allows the user to maintain multiple windows showing different views of the model space (i.e., views of the form, function, and behaviour spaces) as well as views of results from multiple application modules (i.e., views of results from the model construction and model simulation modules). Changes to the model space are automatically updated in all open views. Finally, a range of GUI tools for model creation, editing and manipulation are provided, including: selection, cut, paste, rotation, dragging, Boolean operations, blending of edges, and importing existing models.

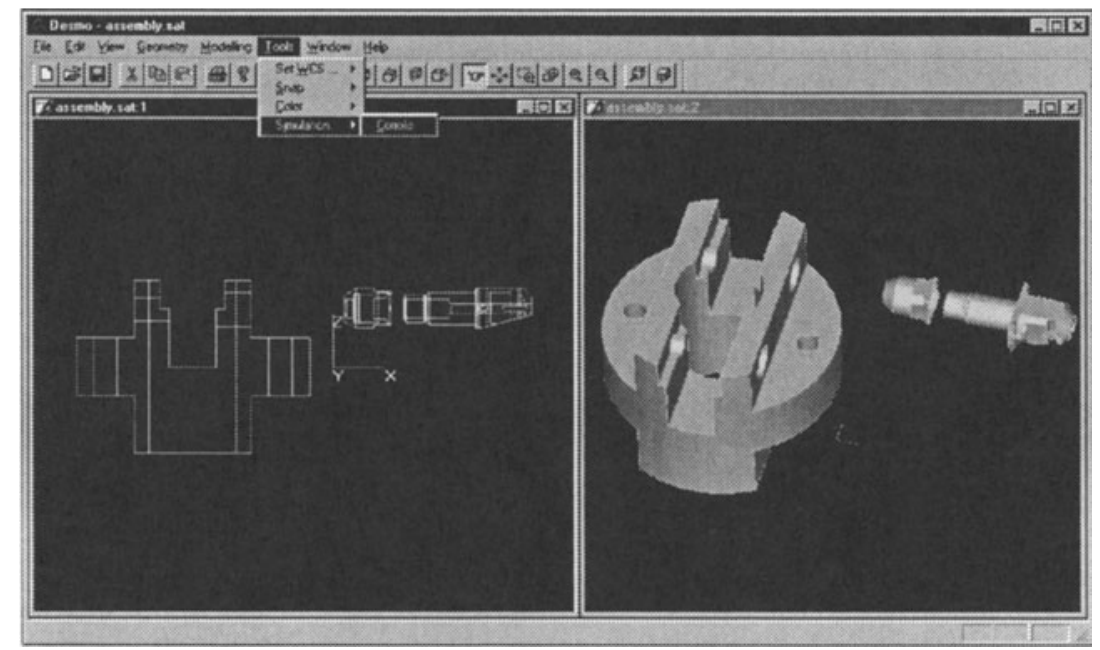

Figure 4. A graphical interface for model construction 


\subsection{Simulation Modules}

The difficulty of creating an analytical model of a design from the knowledge available in existing design modelling frameworks (i.e., solidmodelling information) remains an obstacle to the use of simulation for exploring concepts in early design. Our design modelling kernel supports simulation by representing the high-level knowledge about design intent needed to guide the process of abstracting from a geometric model to an analytical model. The knowledge is modelled by the designer as part of the design process because the designer is able to compose the design model in terms of function and behaviour as well as form.

Simulation modules have two roles: they provide an analysis of behaviour to allow evaluation of a completed design, and they provide the capability to increase the scope of the conversation between the design and the designer through interaction with an evolving design concept's behaviour. These two roles require a different degree of linkage between the module in the design modelling environment that handles model creation, and the module(s) that provide the simulation functionality.

Most modelling systems support only a uni-directional flow of information from the design data-model to the simulation data-model. In this case, the high-level knowledge within the design data-model facilitates the process of abstracting from the design model to the simulation model. The second type of linkage requires an interface that supports a bidirectional flow of information between the construction and simulation modules. The information flow is bi-directional because the simulation module must be able to retrieve current state information about a design from the design data-model and to effect changes to the design model based on the results of a simulation. The two scenarios for linking the design construction and simulation modules are shown in Figures 5 and 6 below.

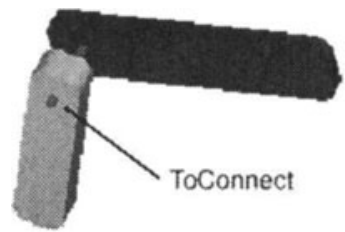

Construction Module

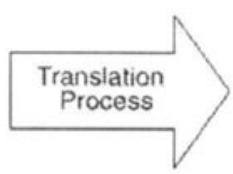

RigidBody 1
RigidBody2

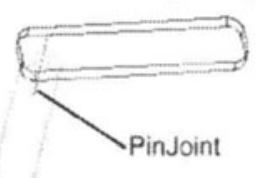

Simulation Module 


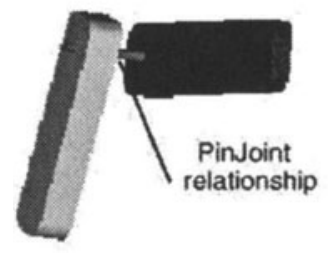

Construction Module

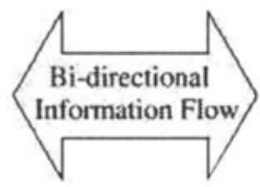

Figure 6. Supporting design within an active simulation

The data-model itself cannot guarantee the consistency of the knowledge structures created by the designer. Knowledge structures can be inconsistent and/or incorrect in a number of ways: a causal relationship may be incorrect (e.g., a behaviour does not cause another behaviour), an implementation relationship may be incorrect (e.g., a part cannot achieve an intended behaviour), and an accumulation may be incorrect (e.g., a set of functions does not contribute to an overall function). It is expected that this type of analysis, to verify consistency, will be performed by one class of simulation module.

The rational for separating consistency checking from the basic modelling framework is that this arrangement most closely resembles the situation that occurs during mental simulation (unaided by computer). For example, in the case where a designer is working without the aid of a computer, a design concept that is generated remains untested (and essentially unproven) until it is physically realized. There the designer cannot know that the form of the design will behave as intended or that the given behaviour will achieve the intended function. The designer gains confidence in the correctness of the design as simulations at successively lower levels of abstraction are performed - from mental simulations through to testing on the final physical prototype. Similarly within a computational design environment, simulation is needed to verify the correctness of the design model.

\subsection{A Data-model for Design Artefact Knowledge}

Our data-model explicitly represents the functional and behavioural knowledge that defines the intent behind the physical embodiment of a design. Our design data-model represents the structure of the knowledge and the relationships between these structures, which are the basis of design 
intent. These structures and relationships are normally generated as part of the process in early design.

A design data-model is an improvement over existing data-models based on solid modelling because in the early stages of conceptualisation a design feature may exist in the mind of the designer as an abstract notion of desired functionality or behaviour. Solid modelling representations force the designer to commit to one physical interpretation of these concepts. Design concepts are generated in terms of function and behaviour as well as form. Our data-model allows the designer to model the intended function and behaviour of the design through composition of design features. Another benefit of a data-model, which is not based on a representation of solids, is more strategic. Specifically, knowledge about design intent is useful in all downstream activities that involve abstraction of the design model. By extending the representation of the design model to include intent as well as form, we provide the basic infrastructure to support knowledge intensive downstream processes.

In our data-model, a design model is composed of concepts (Concept_Entity). Concepts have three aspects, or phases that define the concept in terms of its intended function, behaviour and form. These are defined in the data-model as the Function_Entity, Behaviour_Entity, and Form_Entity object classes. Because the designer concepts design to address specific issues or requirements, concepts can be thought of as being analogous to design features. Like design features, concepts may address multiple functions. The different functions may be associated with different domains (e.g., structural, thermal, kinematic domains). Since functions are realized through behaviours, concepts may therefore also possess multiple behaviours of interest from more than one domain.

\subsubsection{Function Entity}

Our representation for function has three requirements: it cannot be dependent on the pre-existence of a model of behaviour or form, it should relate to models of behaviour, and the individual function structures should be composable. Previous work in function representation has addressed some, but not all of the three above requirements simultaneously. Chandrasekaran's Functional Representation (Chandrasekaran, 1994) represents active functions in terms of state changes. However, little work has been done on its applicability to passive functions such as that of a column supporting a structural load. Furthermore, the Functional Representation framework models function at the device level and do not include the idea of functional composition. (Hodges, 1992) addresses part of 
the problem of creating more complex functions by assembling simpler functional entities, for certain classes of devices. He classifies functions in terms of a hierarchy of general machine elements like linkage, lever, wheelaxle, and gear. However, because his scheme defines functions as sequences of behavioural primitives, the approach requires that a behavioural model of the design be created prior to the functional model.

Our design data-model for representing functional knowledge assumes that, at a high level of abstraction, relationships can be specified between functions and between functions and concepts. At lower levels of abstraction, the interaction between concepts may be specified to occur through regions of space defined by abstractions of form. Because these regions of space are abstractions of physical form, they are not necessarily manifold. Yet, even at this relatively high level of geometric abstraction, sufficient information has been specified to transform a strictly functional concept into one that includes an aspect of behaviour. For example, the control function of a component that allows a chair-back to tilt relative to the seat may act on the chair-back through a shared axis of rotation. The axis of rotation might be represented by a topological entity such as wire or edge. As such, an axis of rotation would be part of the form aspect of a concept. The specification of the axis of rotation assumes a behaviour (i.e., revolute) that is intended to achieve the controlling function. For this reason, the function class object maintains only direct links to other function objects or concepts.

At a high level of abstraction, relationships between functions are of two types: hierarchical and temporal. Hierarchical relationships are maintained through parent and children descriptors. Temporal relationships are maintained through the antecedent and subsequent descriptors. Relationships between functions at lower levels of abstraction, where form comes into play, occur through the function's associated behaviour instance. We will elaborate on these regions of interaction later in this chapter when we discuss representation of the behaviour entity.

\subsubsection{Behaviour Entity}

A Behaviour Entity object models a part of the intended behaviour of a particular design concept. It is possible for a design concept to have multiple behaviours belonging to different domains and appearing under different conditions. A behaviour entity is meant to support an arbitrary description of behaviour. Like the Function Entity, a model of behaviour should not be dependent on a pre-existing functional model of the design. This allows the design to move forward in a heterogeneous manner. However, since designed behaviour is the mechanism by which a function is 
achieved, behaviour's relationship to its associated function needs to be maintained. In mechanical artefacts, behaviour is closely tied to form. We need to be able to identify the parts of the form that are associated with a behaviour. These parts may or may not be physically realizable depending on the current level of development of the design. Behaviour is described both in terms of state transformation between initial and final states, as well as in terms of information flow given by the direction of the relationships joining the different entities within the concept/relationship structure.

A behaviour interacts with external entities (other concepts) through its form phase. The interface for this interaction within the behaviour object is provided by the Region Entity object. Instances of the Region object describe the source and destination regions of interaction for a behaviour. The distinction between source and destination regions allows a causal relationship to be created in those cases where a direction of flow exists in the model of the behaviour. For example, Figure 7 below shows a revolute kinematic behaviour entity connecting two links. If link-A is driving link-B, the source region for the revolute behaviour is the interface between link-A and the revolute. Depending on the level of abstraction, the region of interaction may be a common axis of rotation or the mating faces between the hole in link-A and the surface of the revolute. Similarly, the destination region is the interface between link-B and the revolute.

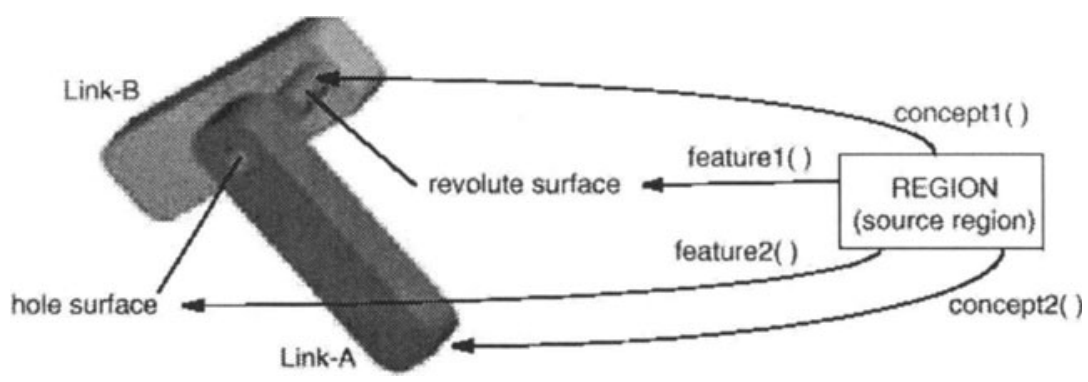

Figure 7. Link-A driving Link-B through a revolute behaviour entity

The Behaviour Entity also maintains a description of its initial and final states using a State Entity object. A State Entity contains the set of parameter and value pairs used to describe a behaviour. Parameter/value pairs are stored as a linked list of instances of classes derived from a common base class, which provides the basic naming and linking methods. The derived classes enable multiple types of parameters to be stored on the same list. The each State Entity has a pointer back to its owning behaviour. 


\subsubsection{Form Entity}

The Form aspect of a concept is based on a boundary-representation topological model. The B-rep model specifies the spatial relationships of geometric elements used to represent the boundary of an object. Using the topological elements of the B-rep model, the geometry of physical and nonphysical features within the design can be represented within the same model. Examples of non-physical design features of interest are:

- centre-lines - e.g. for locating centres of holes, axes of rotation,

- paths - e.g. for defining motion paths, sweep,

- faces - e.g. for indicating contact surfaces or regions of interaction.

The top-level object within the form aspect of a concept is the Body Entity. Starting from the root body, the B-rep hierarchy can be traversed to locate the position of all the geometric features of the model in space.

\section{DEMONSTRATION OF CONCEPT}

The construction of a revolute joint connecting two links is used to illustrate how the functional and behavioural model can be built alongside the geometric model of a design concept. The process begins with the designer creating three concepts: one for the revolute behaviour and two for the links. (See Figure 8.) Initially, the concept entities have no form, behaviour, or function associated with them. The designer is free to develop each aspect of a design concept in a heterogeneous manner. As shown in Figure 8, the designer may choose to refine the form aspect of the link concepts, without refining that of the third concept entity. One possible reason for this may be that the designer is primarily concerned with the length of the links and wishes to ensure that they do not interfere with other objects in the surrounding area. At this point in the process, the designer is not concerned with the form of the design concept connecting the links. Rather, he/she is solely interested in the connecting concept's behaviour as a kinematic pair. The designer adds a kinematic behaviour to the third concept entity (Figure 9) and further defines its relationship with the two links.

Specifically the designer identifies the lines-lines for axes of rotation on the two links as the source and destination feature regions for the kinematic pair, and adds a Control function to the concept entity. At this point, the designer is able to start a kinematic simulation tool (Baraff, 1995) attached to the modelling environment and to load the design model into the simulation module. The simulation module creates a specialized simulation model from the design data-model using the knowledge about function and behaviour, which is represented along side the form elements. 


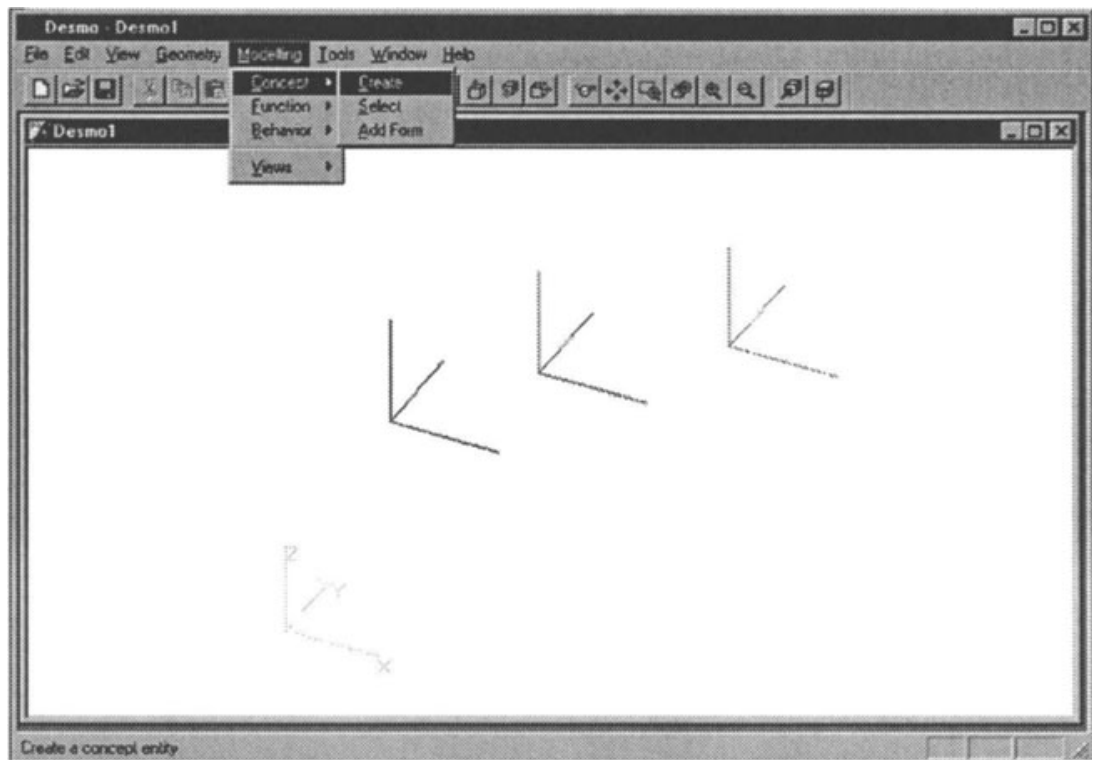

Figure 8 . Three concept entities, each represented by a local coordinate frame

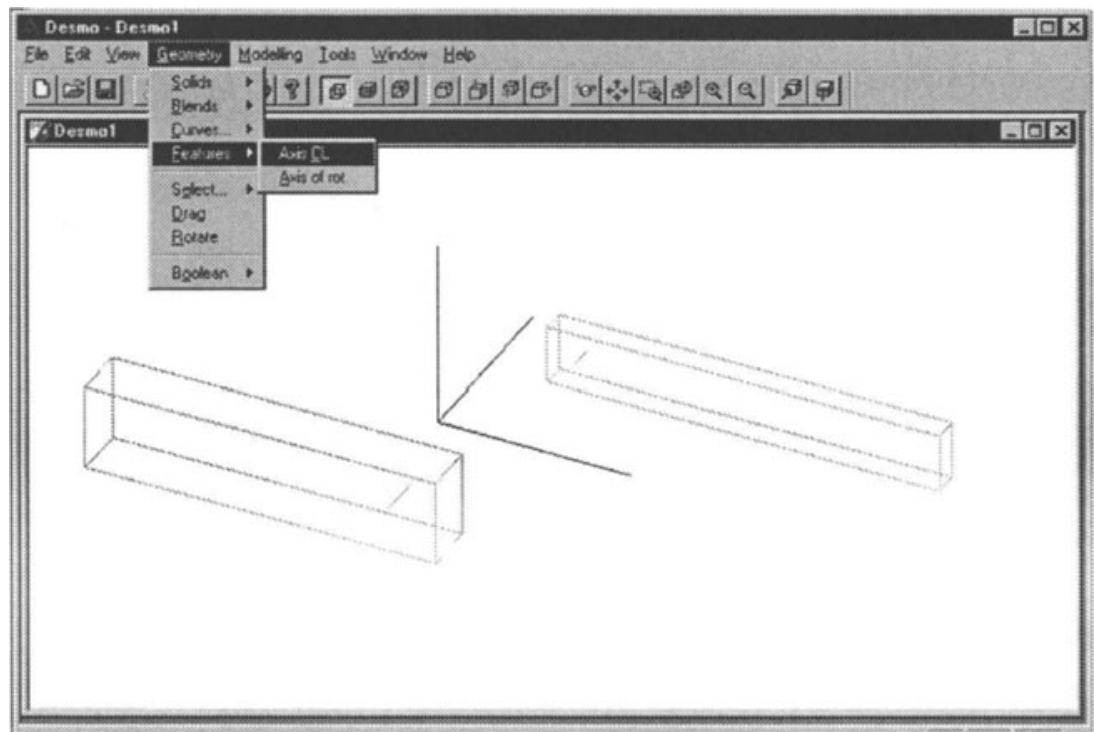

Figure 9. Form refinement of the two concepts representing links 


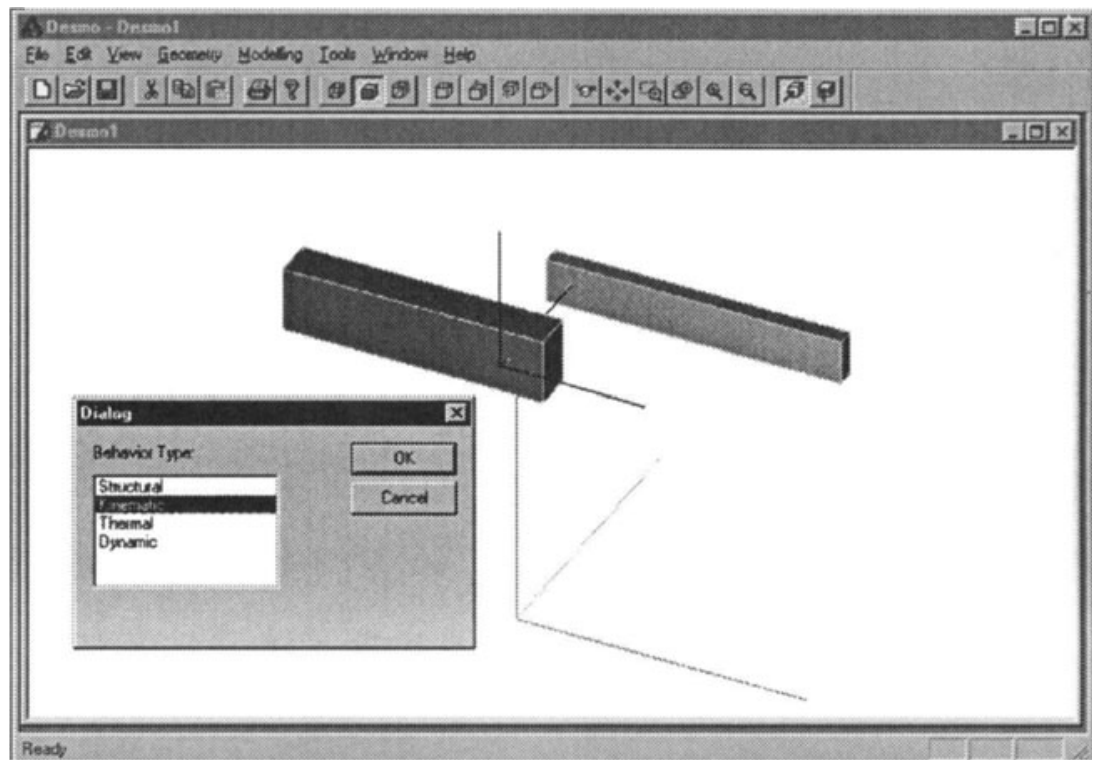

Figure 10. Adding kinematic behaviour to the third concept and making relationships to the link entities

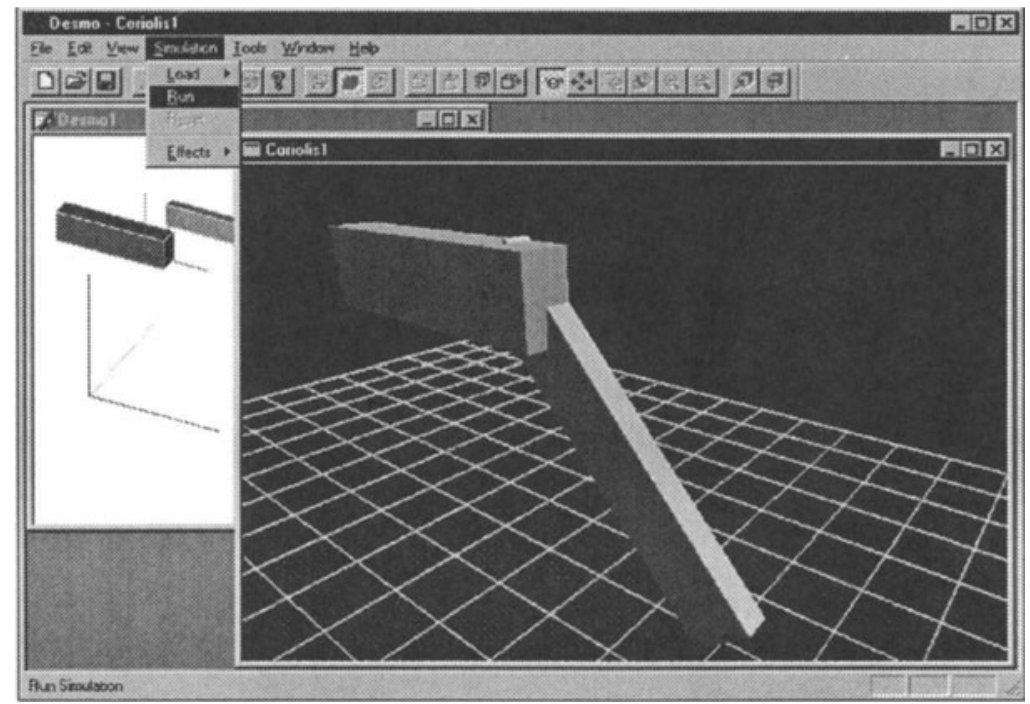

Figure 11. Running a Coriolis simulation 


\section{CONCLUSIONS}

We note that among existing computer-aided engineering tools, the purpose of CAD tools is to construct the geometry that is used as input to downstream analysis tools. For this reason, the capabilities of current CAD tools are not suited for exploring concepts in the early stages of design, and their underlying data-models of the design artefact do not support the explicit representation of knowledge that describes the function and behaviour of a design. To address this deficiency we have developed a datamodel and an architecture for a computational design environment that support the modelling of explicit high-level knowledge about design artefact function and behaviour and the reuse of this knowledge by downstream processes.

A reflective-interactive methodology for design leads to a set of requirements for design tools that are not met by current CAD systems. These requirements include the ability to model knowledge that describes the design context, a closer relationship between the construction and simulation of a design model, and a relaxation of the manner in which design models are constructed. The reflective-interactive methodology offers the opportunity for, and a mechanism through which, design context can be captured as part of the normal process of designing. The framework for capturing and representing the functional and behavioural knowledge consists of a data-model. The basis for the methodology is a synthetic and exploratory model of reasoning in design. The synthetic model explains the process for formulating an initial problem statement, it is better suited to early design when the problem and the solution space are ill-defined, and it fits in within the broader theory of knowledge building as the primary product of the design process.

\section{REFERENCES}

Allen, C., (1989) "Situated Design," Master's thesis, Design Studies, Carnegie-Mellon University, Pittsburgh, PA.

Baraff, D., (1995) "Interactive Simulation of Solid Rigid Bodies," IEEE Computer Graphics and Applications, Vol. 15, No. 3, pp. 63-75.

Baya, V. and Leifer, L. J., (1994) "A Study of Information Handling Behavior of Designers During Conceptual Design," Design Theory and Methodology - DTM'94, American Society of Mechanical Engineers, Minneapolis, MN, pp. 153-168.

Chan, B., (1998) Supporting Conceptual Design: A Methodology and Infrastructure for CAD, PhD dissertation, Civil Engineering, Carnegie Mellon University, Pittsburgh, PA.

Chandrasekaran, B., (1994) "Functional Representation: A Brief Historical Perspective," Applied Artificial Intelligence, Vol. 8, pp. 173-197. 
Dorst, K. and Dijkhuis, J., (1995) “Comparing Paradigms for Describing Design Activity," Design Studies, Vol. 16, No. 2, pp. 261-274.

Faltings, B., (1992) "Supporting Creativity in Symbolic Computation," Proceedings of the 2nd International Conference on Computational Models of Creative Design, J. S. Gero and F. Sudweeks, ed., University of Sydney, Sydney, Australia, pp. 191-206.

Ganeshan, R., Garrett, J. and Finger, S., (1992) "A Framework for Representing Design Intent," Design Studies, Vol 15, No 1, pp. 59-84.

Gero J. and Kazakov V. A., (1996) "An Exploration-Based Evolutionary Model of a Generative Design Process," Microcomputers in Civil Engineering, Vol. 11, pp. 211-218.

Hakim, M., (1993) Modeling Evolving Information about Design Engineering Products, PhD dissertation, Civil Engineering, Carnegie Mellon University, Pittsburgh, PA.

Hodges, J., (1992) "Naive Mechanisms," IEEE Expert, pp. 14-27.

Poon, J. and Maher, M. L., (1997) "Co-evolution and Emergence in Design," Artificial Intelligence in Engineering, Vol. 11, No. 3, pp. 319-327.

Reddy, J. M., (1996) Building and Reuse of Artifact Theories: A View of Design and its Implications for Computational Environments, $\mathrm{PhD}$ dissertation, Civil \& Environmental Engineering, Carnegie Mellon University, Pittsburgh, PA.

Reddy, J. M., Chan, B. and Finger, S., (1996) "Patterns in Design Discourse: A Case Study," Knowledge Intensive CAD, Volume 1, Chapman \& Hall, London, 1996, pp. $265-$ 283.

Schön (1983) Schön, D. A., The Reflective Practitioner, Harper-Collins, New York.

SEED (1997) SEED, "SEED: A Software Environment to Support Early Phases in Building Design," International Journal for Design Computing.

Shaw et al. (1989) Shaw, N. K., Bloor, M. S. and de Pennington, A., "Product Data Models," Research in Engineering Design, Vol. 1, No. 1, pp. 43-50.

Smithers and Troxell (1990) Smithers, T. and Troxell, W., "Design is Intelligent Behavior, But What's the Formalism?" Artificial Intelligence for Engineering Design, Analysis and Manufacturing, Vol. 4, No. 2, pp. 89-98.

STI (1997) STI, "ACIS 3D Toolkit - Modeling Primer, Version 3," Spatial Technologies, Inc., Boulder, CO.

Subrahmanian, E., Konda, S. L., Levy, S. N., Reich, Y. and Westerberg, A. W., (1993) "Equations Aren't Enough: Informal Modeling in Design," Artificial Intelligence in Engineering Design, Analysis and Manufacturing, Vol. 7, No. 4, pp. 257-74.

Tanir, O., Agarwal, V. K. and Bhatt, P. C. P., (1993) "System Design Exploration in a Specification Driven Simulation Environment," Proceedings of the 1993 European Simulation Symposium, Verbraeck, A.; Kerckhoffs, E. J. H., ed., Society for Computer Simulation, Delft, Netherlands, pp. 591-596.

Tomiyama, T., Kiriyama, T., Takeda, H., Xue, D. and Yoshikawa, H., (1989) "Metamodel: A Key to Intelligent CAD Systems," Research in Engineering Design, Vol. 1, No. 1, pp. 19-34.

Woo, M., Neider, J. L. and Davis, T. R., (1997) OpenGL Programming Guide, 2nd Edition. A-W Developers Press. 